\title{
Um Novo Método Euleriano-Lagrangeano para Aproximação de Leis de Conservação
}

S. MANCUSO ${ }^{1}$, F. PEREIRA ${ }^{2}$, G. de SOUZA ${ }^{3}$, LABTRAN, Departamento de Modelagem Computacional, IPRJ, UERJ, 28630-050 Nova Friburgo, RJ, Brasil.

\begin{abstract}
Resumo. Apresenta-se aqui um novo método de alta resolução para leis de conservação escalares que utiliza de uma metodologia do tipo REA ("Reconstruction", "Evolve", "Average"). O método combina a estratégia de [5] para as etapas de "Reconstruction" e "Average" com um método lagrangeano localmente conservativo (veja [3, 1]) para a etapa de "Evolve". Estabelece-se a relação entre o novo esquema e uma estratégia puramente euleriana. O novo esquema foi utilizado para aproximar numericamente leis de conservação escalares em uma dimensão espacial e produziu resultados bastante satisfatórios quando comparados com o esquema central de segunda ordem de [5]. O novo esquema foi aplicado na aproximação numérica das equações de Burgers e Buckley-Leverett, sendo esta última utilizada com o intuito de avaliar o desempenho do método em escoamentos bifásicos em meios porosos.
\end{abstract}

\section{Introdução}

Na modelagem de fenômenos físicos por leis de conservação hiperbólica, freqüentemente aparecem não-linearidades que dificultam o desenvolvimento de métodos que façam o cálculo preciso da solução numérica. Estas soluções não devem apresentar oscilações espúrias ou difusão numérica acentuada. Neste sentido, apresenta-se aqui uma metodologia do tipo REA: "Reconstruction", "Evolve" e "Average" que além das características desejáveis acima, não faz uso de soluções de problemas de Riemann e é baseada em uma estratégia euleriano-lagrangeana. A etapa de "Reconstruction" caracteriza-se por uma aproximação linear por partes. A metodologia foi aplicada em duas equações: a equação de Burgers, e a equação de Buckley-Leverett, esta última usada na modelagem de escoamentos bifásicos em reservatórios de petróleo.

\section{O novo Método Euleriano-Lagrangeano}

A seguir é apresentada a construção do novo esquema euleriano-lagrangeano, baseado no desenvolvimentos de [3, 4]. O método utiliza evoluções para malhas deslo-

\footnotetext{
${ }^{1}$ smancuso@iprj.uerj.br

${ }^{2}$ pereira@iprj.uerj.br

3 ev_grazione@iprj.uerj.br
} 
cadas. Considera-se uma lei de conservação hiperbólica em uma dimensão espacial, como a apresentada pela equação (2.1), onde $f$ é a função de fluxo

$$
u_{t}+f(u)_{x}=0,
$$

ou na sua forma divergente, como a equação

$$
\nabla_{t, x} \cdot\left(\begin{array}{c}
u \\
f(u)
\end{array}\right)=0 \quad x \in \mathbb{R}, \quad \text { com condição inicial } \quad u(x, 0)=u_{0}(x) .
$$

\subsection{Etapas "Average" e "Reconstruction"}

Uma discretização espacial em malha uniforme de comprimento $\Delta x$ para cada célula é aplicada à equação (2.1). Na malha original (deslocada), $x_{j}\left(x_{j-1 / 2}\right.$ ou $\left.x_{j+1 / 2}\right)$ representará o centro (vértice) da j-ésima célula ( $j$ é um inteiro) e $x_{j-1 / 2}$ e $x_{j+1 / 2}$, os seus vértices (centros). A Figura 1 ilustra a construção de malhas. Define-se $U_{j}$ como a discretização constante por partes de $u$ :

$$
U_{j}(t)=\frac{1}{\Delta x} \int_{x_{j-1 / 2}}^{x_{j+1 / 2}} u(x, t) d x .
$$

As variáveis discretizadas calculadas no tempo $t^{n}$ são denotadas acrescentandolhes o índice superior $n$, um inteiro positivo. O n-ésimo intervalo de tempo é denotado por $\Delta t^{n}$ (o instante $t^{n}$ é a soma dos intervalos anteriores). Considerando a formulação fraca de (2.1) (ver [2]) e a discretização (2.3 pode-se calcular o valor de $U$ em uma determinada região através da equação (2.4) no instante $n+1$,

$$
\frac{U_{j}^{n+1}-U_{j}^{n}}{\Delta t}=-\frac{\Delta f_{j}^{n}}{\Delta x} \quad \Rightarrow \quad U_{j}^{n+1}=U^{n}-\frac{\Delta t \Delta f_{j}}{\Delta x}
$$

onde

$$
\Delta f_{j}=f_{j r}-f_{j l} \quad \text { sendo }\left\{\begin{array}{l}
f_{j r}=\int_{t^{n}}^{t^{n+1}} f\left(u\left(x_{j+1 / 2}, t\right)\right) d t \\
f_{j l}=\int_{t^{n}}^{t^{n+1}} f\left(u\left(x_{j-1 / 2}, t\right)\right) d t
\end{array} .\right.
$$

A idéia do método é aproximar $f_{j r}$ e $f_{j l}$ por $\hat{f}_{j r}$ e $\hat{f}_{j l}$, onde $\hat{f}_{j r} \Delta t$ e $\hat{f}_{j l} \Delta t$ são os fluxos associados a cada fronteira, como mostrado na seção (2.2.). A equação abaixo apresenta o esquema usando malha deslocada:

$$
U_{j+1 / 2}^{n+1}=\frac{1}{2}\left(U_{j}^{n}+U_{j+1}^{n}\right)-\frac{\Delta t \Delta f_{j+1 / 2}}{\Delta x},
$$

Com alguns ajustes, pode-se aumentar a precisão do método. Primeiro, substituise a função constante por partes $U(x, t)$ por uma função linear por partes $L(x, t)$. Para tanto, obtém-se uma inclinação $U_{j}^{\prime}(t)$, associada à célula de centro $x_{j}$. A derivada espacial numérica é obtida através de um tipo de MinMod (Módulo mínimo; ver [2]), apresentado pela equação:

$$
\frac{\Delta U_{j}(t)}{\Delta x}=\alpha \operatorname{modmin}\{a, b, c\} a /|a|,
$$


onde

$$
a=\frac{U_{j}(t)-U_{j-1}(t)}{\Delta x}, b=\frac{U_{j+1}(t)-U_{j-1}(t)}{2 \Delta x}, c=\frac{U_{j+1}(t)-U_{j}(t)}{\Delta x}
$$

e min é a função mínimo e $\alpha$ é um coeficiente de ajuste para o MinMod. A Figura 1 também mostra a reconstrução linear.

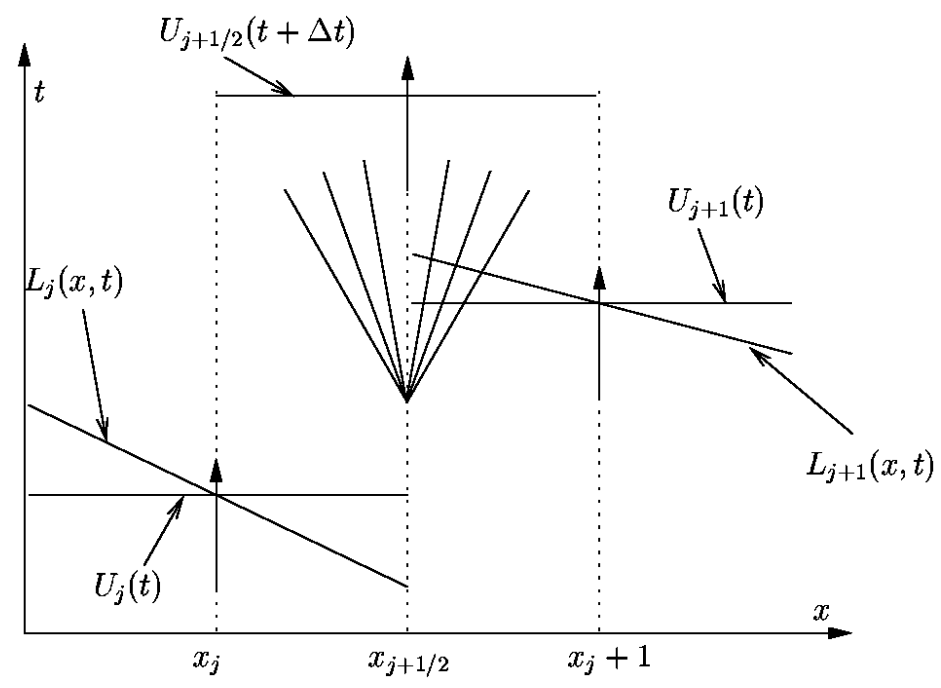

Figura 1: Evolução para malha deslocada e reconstrução linear.

Constrói-se agora, usando os valores $\Delta U_{j}(t)$, a discretização linear por partes mostrada abaixo, que mantém a conservação local:

$$
L_{j}\left(x, t^{n}\right)=U_{j}^{n}+\left(x-x_{j}\right) \frac{\Delta U_{j}^{n}}{\Delta x}, \quad x_{j-1 / 2}<x \leq x_{j+1 / 2},
$$

De acordo com (2.9), pode-se escrever (2.6) como

$$
U_{j+1 / 2}^{n+1}=\left[\int_{x_{j}}^{x_{j+1 / 2}} L_{j}(x, t) d x+\int_{x_{j+1 / 2}}^{x_{j+1}} L_{j+1}(x, t) d x-\Delta t \Delta f_{j+1 / 2}\right] \frac{1}{\Delta x} .
$$

Uma vez determinados os valores de $U$ no instante $t+\Delta t$, repete-se o procedimento partindo, agora, dos centros das células da malha deslocada. Neste caso, considerando que a malha deslocada está definida no tempo $t$, a função $U$ definida novamente na malha original é calculada por

$$
U_{j}^{n+1}=\frac{1}{2}\left(U_{j-1 / 2}^{n}(t)+U_{j+1 / 2}^{n}(t)\right)-\frac{\Delta t \Delta f_{j}}{\Delta x} .
$$

A expressão acima foi obtida seguindo-se um raciocínio análogo ao utilizado para derivar (2.6) e usando os resultados do passo de cálculo anterior. Repete-se o 
esquema até terminar simulação. A metodologia que utiliza discretização constante por partes foi denominada de FLCELM ("Forward Locally Conservative Eulerian Lagrangian Method"), enquanto o método que usa a reconstrução linear foi chamado de FLCELM-R (FLCELM with "Reconstruction").

\subsection{Cálculo dos fluxos nas fronteiras}

Os valores de $u$ no futuro necessários para o cálculo de $\Delta f \Delta t$ são obtidos através de uma evolução lagrageana. Usando a estratégia de construção dos tubos no espaçotempo do método $\operatorname{LCELM}([1])$, considera-se a região do espaço-tempo $\mathcal{V}=\mathbb{R} \times$ $\left[t^{n}, t^{n+1}\right]$. Seja $\mathcal{K}$ um intervalo real $[a, b]$. Define-se um subconjunto $\mathcal{D}=\mathcal{D}^{n}(\mathcal{K})$ de $\mathcal{V}$ como segue: para $x=a, b$ constrói-se a solução $y(x, t)$ do seguinte problema de valor inicial, dado por

$$
\left\{\begin{array}{l}
\frac{d y}{d t}=\frac{f(u)}{u}, \quad t^{n} \leq t<t^{n+1} \\
y\left(x, t^{n}\right)=x .
\end{array}\right.
$$

Então, tem-se a equação

$$
\hat{x}^{n+1}(x)=y\left(x, t^{n+1}\right) .
$$

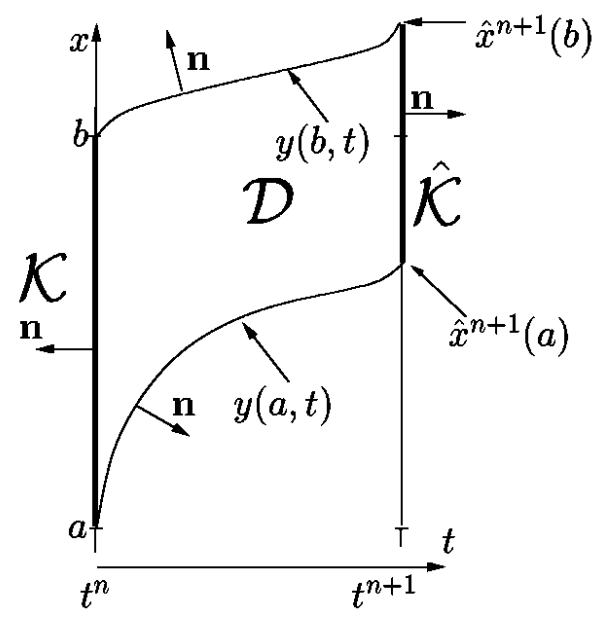

Figura 2: Tubos integrais no espaço-tempo.

Denota-se $\mathcal{K}^{n+1}=\left[\hat{x}^{n+1}(a), \hat{x}^{n+1}(b)\right]$ por $\hat{\mathcal{K}}$. Seja $\mathcal{D}$ o tubo (denominado tubo integral) determinado por $\mathcal{K}, \hat{\mathcal{K}}$,e as curvas integrais (soluções da equação (2.12)). A Figura 2 apresenta os conjuntos $\mathcal{K}, \hat{\mathcal{K}}$ e o domínio $\mathcal{D}$. Pode ser observado que, para $t^{n}$ suficientemente pequeno, a função que associa a cada ponto do domínio espacial $x$ em $t^{n}$ o ponto $\hat{x}^{n+1}(x)$ é bijetiva e, desta forma, esta construção pode ser levada 
a cabo. Denomina-se por $\mathbf{n}(x, t)$ o vetor unitário normal à fronteira de $\mathcal{D}(\partial \mathcal{D})$; ele é ortogonal ao vetor $(u, f(u))^{t}$ nas curvas laterais de $\mathcal{D}$ (ver equação $(2.12)$ ). Portanto, integrando a equação (2.2) sobre $\mathcal{D}$ e usando o teorema da divergência obtém-se a equação

$$
\begin{gathered}
\int_{\mathcal{D}} \nabla_{t, x} \cdot\left(\begin{array}{c}
u \\
f(u)
\end{array}\right) d x d t=\oint_{\partial \mathcal{D}}\left(\begin{array}{c}
u \\
f(u)
\end{array}\right) \cdot \mathbf{n} d L \\
=\int_{\hat{\mathcal{K}}} u\left(x, t^{n+1}\right) d x-\int_{\mathcal{K}} u\left(x, t^{n}\right) d x=0 .
\end{gathered}
$$

Localmente, tem-se

$$
\int_{\mathcal{K}} u\left(x, t^{n}\right) d x=\int_{\hat{\mathcal{K}}} u\left(x, t^{n+1}\right) d x
$$

Considera-se, para a evolução temporal, $U^{n}$ em um instante $t^{n}$ em malha uniforme. Então, são resolvidos os problemas de valor inicial

$$
\left\{\begin{array}{l}
\frac{d y}{d t}=\frac{f\left(U_{j}^{n}\right)}{U_{j}^{n}} \\
y\left(x_{j}^{n}, t^{n}\right)=x_{j}^{n}
\end{array} \quad t^{n} \leq t<t^{n+1} \text { e } j \in \mathbb{Z}\right.
$$

obtendo os pontos $\hat{x}^{n+1}\left(x_{j}^{n}\right), j \in \mathbb{Z}$; estes pontos determinam os vértices de uma malha não uniforme no instante $t^{n+1}$. Assim, constrói-se a malha não uniforme com vértices $\bar{x}_{j-1 / 2}^{n+1}=\hat{x}^{n+1}\left(x_{j}^{n}\right)$ e $\bar{x}_{j+1 / 2}^{n+1}=\hat{x}^{n+1}\left(x_{j+1}^{n}\right), j \in Z$, como é ilustrado pela Figura 3. Considera-se a média

$$
\hat{U}_{j}^{n+1}=\frac{1}{h_{j}^{n+1}} \int_{\hat{\mathcal{K}}} u\left(x, t^{n+1}\right) d x .
$$

De acordo com a equação $(2.15)$, o valor de $\hat{U}_{j}^{n+1}$ em cada intervalo $\left[\bar{x}_{j-1 / 2}^{n+1}, \bar{x}_{j+1 / 2}^{n+1}\right)$, $j \in \mathbb{Z}$, da nova malha é dado por

$$
\hat{U}_{j}^{n+1}=\frac{1}{h_{j}^{n+1}}\left(\int_{x_{j}^{n}}^{x_{j+1 / 2}^{n}} U\left(x, t^{n}\right) d x+\int_{x_{j+1 / 2}^{n}}^{x_{j+1}^{n}} U\left(x, t^{n}\right) d x\right) .
$$

No caso da reconstrução linear por partes

$$
\hat{U}_{j}^{n+1}=\frac{1}{h_{j}^{n+1}}\left(\int_{x_{j}^{n}}^{x_{j+1 / 2}^{n}} L_{j}\left(x, t^{n}\right) d x+\int_{x_{j+1 / 2}^{n}}^{x_{j+1}^{n}} L_{j}\left(x, t^{n}\right) d x\right)
$$

Com uso da reconstrução, os tubos integrais precisam de uma melhor aproximação para os pontos $\hat{x}^{n+1}\left(x_{j}^{n}\right)$. Neste caso, os tubos são construídos por $M$ passos: no primeiro o tubo é construído da forma convencional com um avanço de tempo $\Delta t / M$ usando $U_{j}$ indo de $x_{j}$ até $x_{j, 1}$. Para calcular $x_{j, i+1}$ calcula-se $U_{j, i}$ em $\left(x_{j, i}, t^{n}+i \Delta t / M\right)$ por expansão em série de Taylor no tempo, sobre $L_{j}\left(x_{j, i}, t^{n}\right)$, 


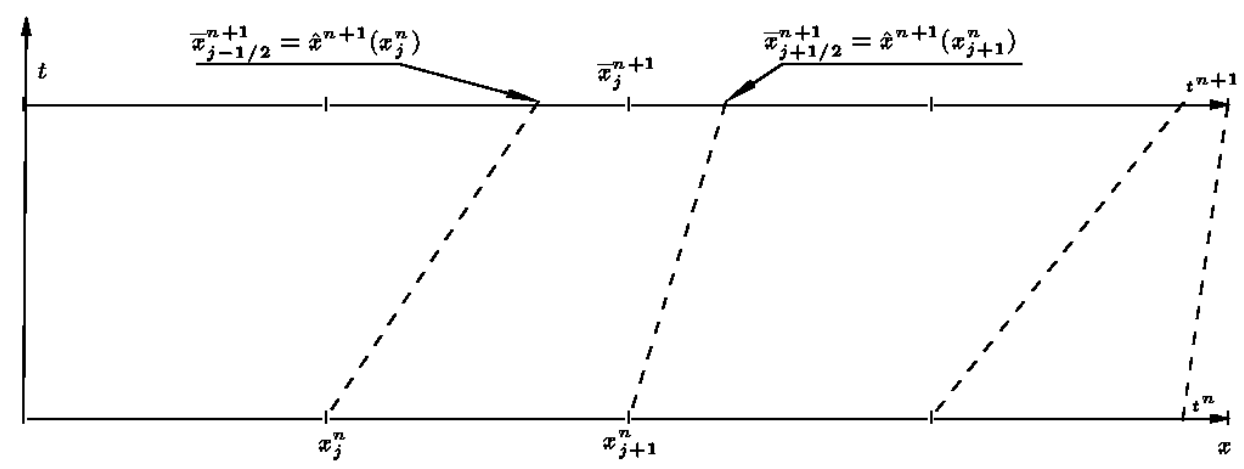

Figura 3: Linhas tracejadas correspondem às tangentes à curva integral no tempo $t^{n}$.

obtendo-se $x_{j, i+1}=x_{j, i}+\frac{f\left(U_{j, i}\right)}{U_{i, j}} \frac{\Delta t}{M}$. Isto é feito enquanto $i<M$ (até completar o $\Delta t$ total permitido). Assim

$$
\hat{f}_{j l}=\frac{1}{\Delta t} \int_{x_{j}^{n}}^{\hat{x}^{n+1}\left(x_{j}^{n}\right)} \hat{U}_{j}\left(x, t^{n+1}\right) d x \text { e } \hat{f}_{j r}=\frac{1}{\Delta t} \int_{x_{j+1}^{n}}^{\hat{x}^{n+1}\left(x_{j+1}^{n}\right)} \hat{U}_{j+1}\left(x, t^{n+1}\right) d x
$$

\subsection{Restrição no passo de tempo}

Para a evolução temporal, toma-se um incremento $\Delta t$ que não permita que a solução dos problemas de Riemann oriundos dos vértices das células atinjam os centros das mesmas. O $\Delta t$ assume dois valores durante a simulação, um para a malha original e outro para a deslocada (faz-se uso do menor tamanho de célula da malha, $h_{m i n}$ ). Utiliza-se a condição:

$$
\max \left\{f_{\text {sup }}^{\prime}-\left(\frac{f(U)}{U}\right)_{\text {sup }},\left(\frac{f(U)}{U}\right)_{\text {inf }}-f_{\text {inf }}^{\prime}\right\} \frac{\Delta t^{n}}{h_{\min }} \leq \frac{1}{2},
$$

onde $\max$ é a função máximo e

$$
\begin{gathered}
f_{\text {sup }}^{\prime}=\sup \left\{f^{\prime}(U) \mid U \in \mathbb{D}_{f^{\prime}}\right\} \text { e } f_{\text {inf }}^{\prime}=\inf \left\{f^{\prime}(U) \mid U \in \mathbb{D}_{f^{\prime}}\right\}, \\
\left(\frac{f(U)}{U}\right)_{\text {sup }}=\sup \left\{\left(\frac{f(U)}{U}\right) \mid U \in \mathbb{D}_{f}\right\} \text { e }\left(\frac{f(U)}{U}\right)_{\text {inf }}=\inf \left\{\left(\frac{f(U)}{U}\right) \mid U \in \mathbb{D}_{f}\right\} .
\end{gathered}
$$

\section{Simulações Numéricas}

Em todas simulações numéricas realizadas utilizou-se uma condição de injeção lateral na fronteira esquerda da malha computacional correspondente ao fluxo relacionado a condição inicial à esquerda do problema de Riemann (detalhes em [3]). A 
função $f$ para a Eq. de Burgers é dada por

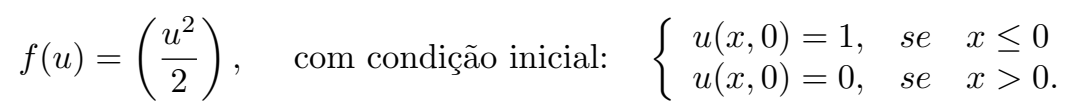

que foi utilizada na equação (2.1). Para a Eq. de Burgers o comprimento da região física foi de $12800 \mathrm{~cm}$ e o tempo de simulação de 15000 s. As Figuras 4 e 5 apresentam um estudo de refinamento de malha e a comparação da solução numérica em 256 células com a solução analítica para o FLCELM, respectivamente.

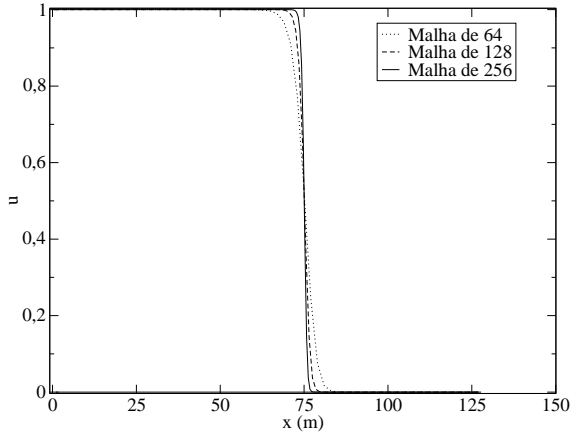

Figura 4: Eq. de Burgers: refinamento de malha (FLCELM).

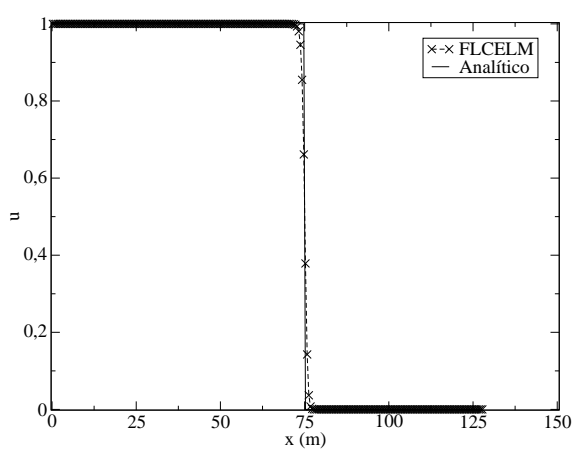

Figura 5: Eq. de Burgers: FLCELM e solução analítica.

As Figuras 6 e 7 apresentam um estudo de refinamento de malha e a comparação da solução numérica em 256 células com a solução analítica para o FLCELM-R, respectivamente, utilizando $M=10$ e $\alpha=1,0$.

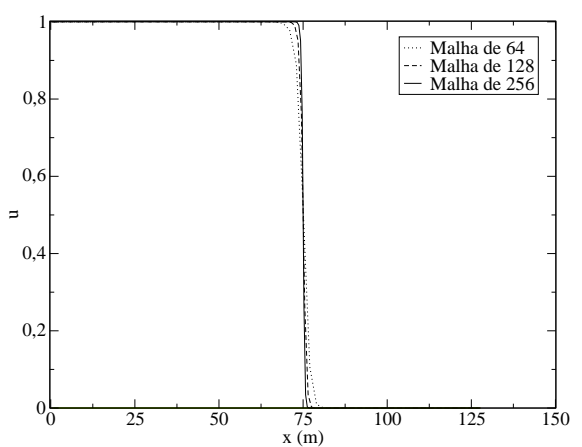

Figura 6: Eq. de Burgers: refinamento de malha (FLCELM-R).

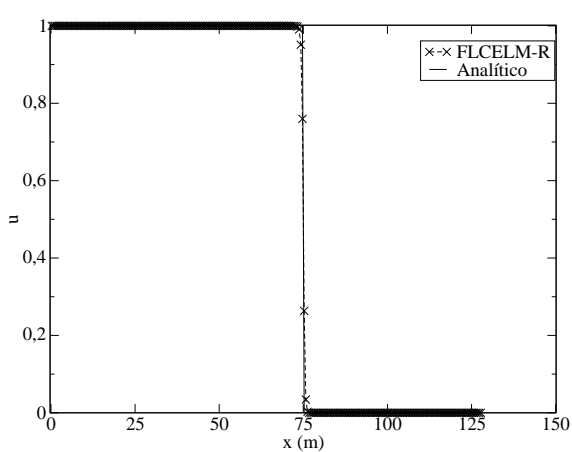

Figura 7: Eq. de Burgers: FLCELM$\mathrm{R}$ e solução analítica.

Claramente a estratégia FLCELM-R produz resultados superiores, sendo menos difusiva quando comparada a FLCELM. Nas comparações com a solução analítica 
nota-se que as simulações conseguiram representar o salto com velocidade de propagação correta e pôde-se observar que em uma malha de 128 células atingi-se um bom nível de convergência numérica.

Para a Eq. de Buckley-Leverett, a função de fluxo $f$ é

$$
f(s)=\lambda_{w}(s) v \quad \operatorname{com} \quad\left\{\begin{array}{l}
\lambda_{w}(s)=\frac{k_{r_{w}}(s)}{\mu_{w} \lambda(s)} \\
\lambda(s)=\frac{k_{r_{o}}(s)}{\mu_{o}}+\frac{k_{r_{w}}(s)}{\mu_{w}} \\
k_{r_{o}}(s)=\left(1-\left(1-s_{r_{o}}\right)^{-1} s\right)^{2} \\
k_{r_{w}}(s)=\left(1-s_{r_{w}}\right)^{-2}\left(s-s_{r_{w}}\right)^{2}
\end{array}\right.
$$

onde $\mathrm{w}=$ água e o=óleo. Para a fase $\alpha(\alpha=w, o)$ tem-se: $\lambda_{\alpha}(s)$, mobilidade; $k_{r_{\alpha}}(s)$, permeabilidade; $s_{r_{\alpha}}$, saturação residual e $\mu_{\alpha}$, a viscosidade. Os demais termos são: $\lambda(s)$, mobilidade total do sistema e $v$, a velocidade do fluido (velocidade de Darcy). Os seguintes valores numéricos foram utilizados nas simulações: $v=8,11235 e^{-5}$; $s_{r_{o}}=0,15 ; s_{r_{w}}=0,20 ; \mu_{o}=10,0 ; \mu_{w}=0,5$. O escalar $s$ representa a saturação de água (massa por unidade espacial) em uma determinada região em um certo instante do escoamento. Para a Eq. de Buckley-Leverett, o comprimento da região física foi de $12800 \mathrm{~cm}$, o tempo de simulação 300 dias e a condição de contorno em $x=0$ é $f(s)=\lambda_{w}(0,85) v$. A condição inicial é uma saturação de 0,85 para $x \leq 0$ e uma saturação de 0,21 para $x>0$. As grandezas físicas estão expressas em CGS. As Figuras 8 e 9 apresentam refinamento de malha e a comparação da solução numérica em 128 células com a solução pelo método NT [5] para o FLCELM-R, respectivamente. O número $M$ de passos utilizados para a construção dos tubos integrais foi igual a 10 e o coeficiente $\alpha$ foi de 1,0 no teste de convergência e 1,5 na comparação com NT.

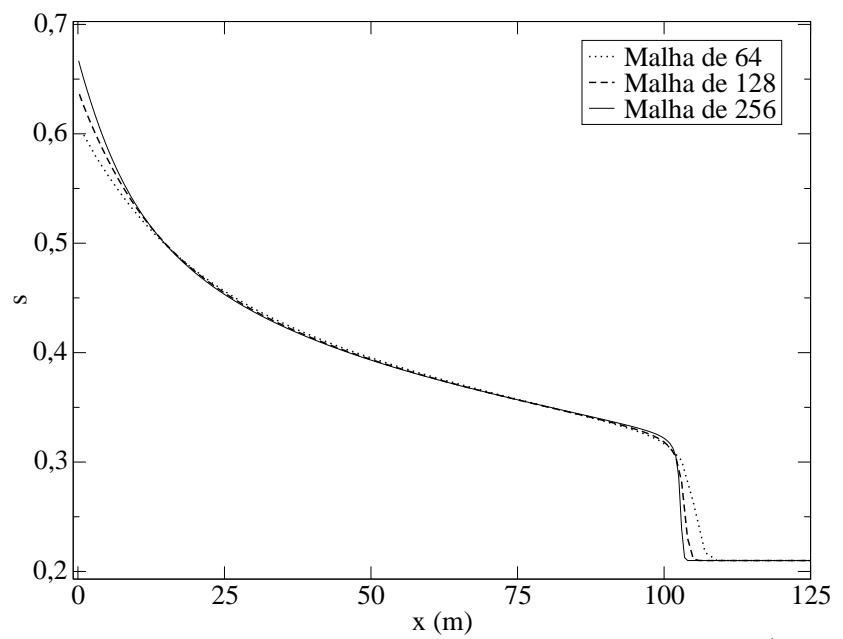

Figura 8: Eq. de Buckley-Leverett: refinamento de malha (FLCELM-R).

Pode-se observar que também para a Eq. de Buckley-Leverett ocorreu convergência sob refinamento da malha computacional. A comparação com o método NT mostra que o choque apresenta velocidade de propagação correta e que o método 


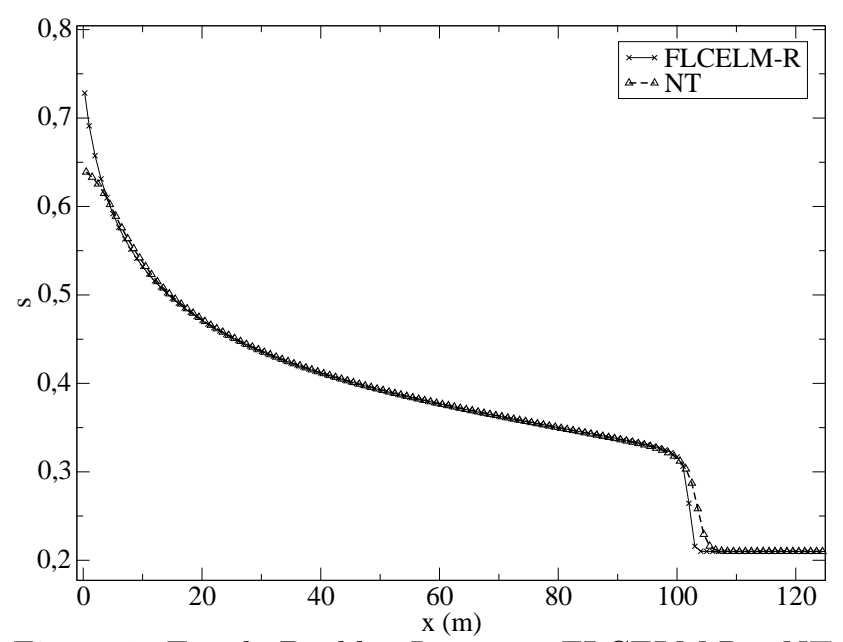

Figura 9: Eq. de Buckley-Leverett: FLCELM-R e NT.

FLCELM-R foi um pouco menos difusivo. Além disso, o comportamento na injeção usando o FLCELM-R está mais próximo da situação física a ser modelada.

\section{Conclusões}

Neste trabalho foi desenvolvido um novo método para a solução numérica de leis de conservação escalares. Duas versões foram testadas: a primeira baseada em uma discretização espacial constante por partes e outra que utiliza uma reconstrução linear descontínua dos valores do escalar $u$. O uso da reconstrução melhorou a precisão do método, diminuindo a difusão numérica. As descontinuidades foram resolvidas de forma precisa e nos experimentos realizados o método convergiu para a solução fisicamente correta. As perspectivas para trabalhos futuros são o desenho de um esquema numérico para a resolução de problemas bi e tridimensionais.

\section{Agradecimentos}

F. Pereira recebeu apoio financeiro dos projetos: CNPq-Edital 05/2004, CT-Petro/ Edital 01/2003, CT-Petro/Edital 016/2005, Edital Institutos do Milênio 01/2005 e Edital 20/2004-Acordo de Cooperação Internacional CNPq/NSF; G. de Souza agradece ao CNPq (Bolsa de Iniciação Científica) e S. Mancuso agradece a CAPES (Bolsa de Doutorado).

\footnotetext{
Abstract. Here is shown a new high resolution method for scalar conservation laws using a REA methodology (Reconstruction, Evolve, Average). It combines the strategy of [5] in the Reconstruction e Average stages with a locally conservative lagrangian scheme (see $[3,1])$ in the Evolve stage. We establish the relation between the new scheme and an eulerian strategy. The new scheme was utilized to solve
} 
scalar conservation laws in one space dimension and produced quite satisfactory results when compared with the method of [5]. We applied the new method in the numerical aproximation of the Burgers and Buckley-Leverett equations. The last one was used to evaluate the perfomance of this method in the simulation two-phase flows in porous media. The extension of the method in two dimensions problems will be discussed.

\section{Referências}

[1] J. Douglas Jr., F. Pereira, L.M. Yeh, A locally conservative EulerianLagrangian numerical method and its application to nonlinear transport in porous media, Computational Geosciences, 4 (2000), 1-40.

[2] R.J. Le Veque, 'Finite Volume Methods for Hyperbolic Problems", Cambridge Texts in applied Mathematics, Cambridge University Press, UK 2002.

[3] S. Mancuso, "Aproximação numérica de leis de conservação por esquemas euleriano-lagrangeanos localmente conservativos." Dissertação de Mestrado, Instituto Politécnico do Rio de Janeiro - Universidade do Estado do Rio de Janeiro, 2004.

[4] S. Mancuso, F. Pereira, Esquemas euleriano-lagrangeanos localmente conservativos para leis de conservação hiperbólicas, in "CD-Rom - Iberian Latin American Congress on Computational Methods of Engineering", 2004.

[5] N. Nessyahu, E. Tadmor, "Non-oscillatory central differencing scheme for hyperbolic conservation laws", Journal of Computational Physics, 87 (1990), 408463. 